\section{Testicular Lymphoma: A Rare Cause of a Testicular Mass}

Sir,

While lymphoma is a well-known malignancy, primary testicular lymphoma is a rare and aggressive cancer which accounts for $1-2 \%$ of all Non-Hodgkin's Lymphoma (NHL) cases. Due to its rarity, there is limited data on the treatment and survival of these patients.

A 65-year male presented with one-month history of insidious onset swelling and pain of his left testicle. He denied local trauma, insect bite, recent travel, dysuria, pyuria or unprotected sexual encounter. Examination showed a firm, approximately average lemon-sized, non-fluctuant, mildly tender swelling in the left scrotum. The testicle could not be appreciated separately from the mass. Rest of the physical examination was unremarkable for hepatosplenomegaly or lymphadenopathy.

Ultrasonography (USG) confirmed a left-sided $7.6 \times 6.1 \times 5.5 \mathrm{~cm}$, solid, vascular testicular mass (Figure 1). Positron emission tomography (PET) scan confirmed a localised disease of the testis with no hypermetabolic activity noted anywhere else in the body. Suspicion of malignancy was considered, and a left testicular radical orchiectomy was performed. Immunohistochemistry (IHC) was diagnostic of diffuse large B cell lymphoma (DLBCL) (Figure 2). He was started on R-CHOP (rituximab, cyclophosphamide, doxorubicin, vincristine, prednisolone) plus intrathecal methotrexate. Till date, the patient has successfully completed and well-tolerated two chemotherapy cycles and a repeat PET scan is planned after 6 cycles.

Testicular DLBCL is not only a challenging entity for oncologists, but also emotionally demanding for the patients due to the complexities of the disease, its location and management. Testicular lymphoma is unique in terms of its location, aggressive nature, and high rate of relapse to contralateral testis/central nervous system (CNS). Over the past few decades, we have come a long way, and now the survival is much better due to advancement in diagnostic modalities. ${ }^{1-3}$ Recently, PET scan has been increasingly used for prognosticating patient with regards to its side of involvement, extent of disease, and risk of relapse. ${ }^{4,5}$

Radiotherapy, addition of rituximab, prophylactic intrathecal chemotherapy, and use of PET/CT scan have certainly improved progression-free survival and overall survival of testicular lymphomas. ${ }^{6}$ PET/CT is a good tool to guide radiation oncologists to determine the radiation field, etc. To reduce the risk of CNS relapse, a CNS directed prophylaxis is usually recommended.

Testicular lymphoma is currently being treated like DLBCL of any other site. However, the risk of relapse to other testis, chances of becoming sterile post-surgery / radiation, and side effects secondary to hypoandrogenism are essential points of discussion with the patient before initiating any treatment. Till now, most of the experiences are only limited to small case series and retrospective studies. Newer diagnostic modalities and immunotherapies have led to early detection and better overall outcome.

\section{CONFLICT OF INTEREST:}

Authors have no conflicts of interest to declare.

\section{AUTHORS' CONTRIBUTIONS:}

KKS: Manuscript writing.

AKM: Literature search.

JO: Critical review, comments and management.

\section{REFERENCES}

1. Sahu KK, Thakur K. Role of positron emission tomography imaging in myeloid sarcoma. Indian J Nucl Med 2018; 33:90.

2. Gautam A, Sahu KK, Alamgir A, Siddiqi I, Ailawadhi S. Extramedullary solitary plasmacytoma: Demonstrating the role of (18)F-FDG PET imaging. J Clin Diagn Res 2017; 1: XD01-XD03.

3. Sahu KK, Gautam A, Ailawadhi S. Re: FDG PET/CT findings of intracardiac myeloid sarcoma. Clin Nucl Med 2017; 42:242-5.

4. Sahu KK, Mishra A, O'shea J. Role of (18)f-fdg positron emission tomography/computed tomography imaging in testicular lymphoma. Mol Imaging Radionucl Ther 2019; 28 : 44-5.

5. Sidhu P, Lin P, Son H, Rosenfeld D, Lin M. Testicular fluorine-18 fludeoxyglucose uptake on positron emission tomo-graphy $\mathrm{CT}$ in patients with lymphoma: Clinical significance and management impact. Br J Radiol 2014; 87: 20140472.

6. Ollila TA, Olszewski AJ. Radiation therapy in primary testicular lymphoma: Does practice match the standard of care? Leuk Lymphoma 2019; 60:523-6.

Kamal Kant Sahu', Ajay Kumar Mishra' ${ }^{1}$, Amos Lal ${ }^{2}$ and James Oshea $^{1}$

${ }^{1}$ Department of Internal Medicine, Saint Vincent Hospital, Worcester, USA

${ }^{2}$ Department of Critical Care and Pulmonary Medicine, Mayo Clinic, Rochester, USA

Correspondence to: Dr. Kamal Kant Sahu, Saint Vincent Hospital, 123 Summer Street, Worcester, MA, USA

E-mail:drkksahu85@gmail.com

Received: July 10, 2019; Revised: July 11, 2019;

Accepted: July 12, 2019

DOI: https://doi.org/10.29271/jcpsp.2020.05.556 